\title{
A review of the efficacy of human $Q$ fever vaccine registered in Australia
}

\author{
Clayton K. Chiu ${ }^{\mathrm{A}}$ and David N. Durrheim ${ }^{\mathrm{B}, \mathrm{C}}$ \\ ${ }^{\mathrm{A}} N \mathrm{SW}$ Public Health Officer Training Program, \\ NSW Department of Health \\ ${ }^{B}$ Hunter New England Population Health, \\ Hunter New England Area Health Service \\ ${ }^{\mathrm{C}}$ Corresponding author. \\ Email: David.Durrheim@hnehealth.nsw.gov.au
}

\begin{abstract}
Background: Q fever remains an important occupational zoonosis in rural Australia. Although Q fever vaccine is recommended in highrisk occupational groups, its availability has been limited in recent years. Method: A literature review of the efficacy of the human $\mathrm{Q}$ fever vaccine registered in Australia was conducted. Results: Seven relevant vaccine efficacy studies were identified but no large double-blind, randomised, placebocontrolled studies have been conducted. Vaccine efficacy has ranged from $83-100 \%$ but limitations of study designs hamper a precise estimate of vaccine efficacy. Conclusion: Despite the shortcomings of efficacy studies, the Q fever vaccine available in Australia has considerable protective benefit in established high-risk environments, particularly of an occupational nature.
\end{abstract}

\section{Background}

Disease burden

$\mathrm{Q}$ fever is a zoonosis caused by Coxiella burnetii. It has been identified in a wide range of wild and domestic animal hosts including arthropods, birds, rodents, marsupials and livestock, but the commonest reservoirs are cattle, sheep and goats. C. burnetii can withstand harsh environmental conditions including desiccation, and are shed in excreta, milk and, particularly, birth products of infected animals. ${ }^{1}$

Humans become infected primarily by inhaling aerosols contaminated by $C$. burnetii. Established higher risk occupations include abattoir and farm workers, and veterinarians. Indirect exposures through a contaminated environment or ingestion of contaminated unpasteurised milk have also been recognised but their contribution is not well quantified. ${ }^{1}$ A Victorian study estimated that the average annual risk of $\mathrm{Q}$ fever among abattoir workers to be $62.6 / 1000$ over the initial 10 years of employment. ${ }^{2}$ Unfortunately, there is currently only a limited understanding of the stratification of risk by nature of exposure, the possible variable pathogenicity of different $C$. burnetii strains, and the disease modulating effect of human immune responses.

Many infected people are asymptomatic or only experience a self-limiting febrile illness. Although Q fever infections generally respond to antibiotic treatment, they may result in significant morbidity, including pneumonia, hepatitis, chronic endocarditis and post-Q fever fatigue syndrome, and occasionally death. Recrudescence may occur, especially in pregnant and immunocompromised patients.

Australia reported between 465 and 757 cases annually between 2000 and 2004. This is likely to be an underestimate because of difficulties in diagnosing $\mathrm{Q}$ fever and the current passive disease surveillance system. Data from the Notifiable Diseases Database in NSW, which reported $47 \%$ of all Australian Q fever cases in 2004, indicated that many notified cases still occurred among known high-risk groups. In 2004 and 2005 (to 22 November), 41\% of cases with an identified occupation were farm workers and $13 \%$ were abattoir workers, meat workers, livestock handlers or veterinarians.

\section{Disease control}

A national vaccination program against $\mathrm{Q}$ fever that initially targeted abattoir workers, and subsequently also shearers and people employed on sheep and cattle farms, was conducted in Australia between 2001 and 2004. The program was evaluated by the National Centre for Immunisation Research and Surveillance for the Australian Government Department of Health and Ageing, but the resulting report has not yet been published.

Although environmental containment measures, including environmental cleaning and use of personal protective equipment, are often recommended as adjuncts to vaccination for $\mathrm{Q}$ fever control, no specific studies of the effectiveness of these measures have been conducted.

\section{Human Q fever vaccines}

Three types of vaccine have been proposed for providing human protection against $\mathrm{Q}$ fever: the attenuated live vaccine (produced and trialled in Russia but subsequently 
abandoned because of concern about its safety); chloroform-methanol residue extracted vaccine or other extracted vaccines (trialled in animals but not humans); and the whole-cell formalin-inactivated vaccine, which is considered acceptably safe for humans. ${ }^{3}$ Australia is the only developed country that manufactures this latter $\mathrm{Q}$ fever vaccine on a commercial basis and it has been available in Australia since 1989. Ongoing Q fever vaccine availability in Australia became uncertain during late 2005.

\section{Methods}

A search of the Medline and Embase databases was conducted to identify all published English language articles on the efficacy of the formalin-inactivated Henzerling strain phase I vaccine in humans, using subject headings and keywords: Q fever, Coxiella burnetii, Henzerling, 'Q-vax', vaccine, vaccination and immunisation (and corresponding variants of these keywords). Original reports referenced in articles were also accessed.

\section{Results}

Seven vaccine studies on the efficacy of the formalin-inactivated Henzerling strain phase I vaccine in humans were identified in the literature (Table 1).

One article reported two challenge studies that have been conducted on human volunteers. ${ }^{3}$ The study, performed in the late 1950s, involved 24 subjects and tested an earlier formulation with a three-dose schedule. The reported study conducted between 1966 and 1968 pooled results from two studies conducted in parallel and involved 38 subjects. Both found vaccine efficacy to be $92 \%$, but with broad confidence limits due to the small number of subjects.

A limited, randomised, blinded, placebo-controlled trial of the $\mathrm{Q}$ fever vaccine (using influenza vaccine as a placebocontrol) that utilised a sequential analysis design was conducted in the late 1980s in three Queensland abattoirs with different characteristics. ${ }^{4}$ This study was terminated after 15 months when one-sided $95 \%$ statistical significance was reached between the two study arms, with seven Q fever cases reported in the placebo-control group with no cases in the trial group. The protective efficacy of this vaccine was not estimated in this study. The method of randomisation was not clearly described, nor the intention-totreat principle applied.

A retrospective cohort analysis of a 1998 Q fever outbreak investigation in a southern NSW abattoir during the second phase of a local $\mathrm{Q}$ fever vaccination program provided some useful efficacy information. ${ }^{5}$ Nineteen staff members were vaccinated during the first phase, at least six weeks before the onset of the first outbreak case. No exposure differences were apparent between these
19 people and the 68 susceptible people who were eligible for vaccination during the second phase. Since no Q fever cases occurred in the 19 vaccinated individuals while 37 cases occurred in the 68 unvaccinated individuals, vaccine efficacy was calculated at $100 \%$, although the lower confidence limit cannot be accurately estimated because of absence of cases in the vaccinated individuals.

Three open trials (non-randomised studies without control groups) provided additional insight into the efficacy of the available $\mathrm{Q}$ fever vaccine. ${ }^{6-8}$ Two $\mathrm{Q}$ fever cases were diagnosed among 2553 vaccinated employees from three different abattoirs in South Australia between 1985 and 1990, while 55 cases were reported among 1365 unvaccinated employees during this period, indicating a vaccine efficacy of $98 \%$ with a $95 \%$ lower confidence limit of $92 \%{ }^{6}$ Results may have been affected by volunteer selection bias, uncertain risk exposure estimates and inadequate active $\mathrm{Q}$ fever case ascertainment.

An earlier open trial in three South Australian abattoirs reported four Q fever cases amongst 924 vaccinated workers and 34 cases amongst 1349 unvaccinated workers between June 1981 and January 1983 (Relative risk of vaccinated $(\mathrm{RR})=0.17,95 \%$ CI $0.06-0.48, p=0.00027){ }^{7}$ This study also suffered limitations, as the unvaccinated group included employees who were not enrolled and enrolled volunteers who were not vaccinated, either due to the presence of immune markers or other unstated reasons. The vaccinated group also included a small number of volunteers who had detectable immune markers before vaccination. A greater proportion of unvaccinated workers were deployed in higher risk areas.

A follow-up study of Q fever cases between mid-1981 and 1988 among workers from five different abattoirs in South Australia ${ }^{8}$ included subjects from the study mentioned above. ${ }^{7}$ Some subjects in this study were also included in the study described earlier. ${ }^{6}$ Vaccine efficacy was reported as $100 \%$, with eight $Q$ fever cases among 3532 vaccinated subjects all attributed to exposure before vaccine-induced immunity would have developed. Among the subgroup considered at high risk of exposure, three of 2715 vaccinated subjects developed disease, whereas 52 of 2012 unvaccinated subjects developed disease, a protective efficacy estimate of $96 \%$ (95\% CI 86-99\%, $p<0.0001)$. Analysis of a subgroup of workers from a single abattoir, without immune markers of the disease before contemplating vaccination, revealed two cases of $\mathrm{Q}$ fever among 690 vaccinated individuals and seven cases among 61 unvaccinated subjects, an efficacy estimate of $97 \%$ (95\% CI $88-99 \%, p<0.0001)$.

The only economic study undertaken in Australia on Q fever vaccine predated the completion of the national 


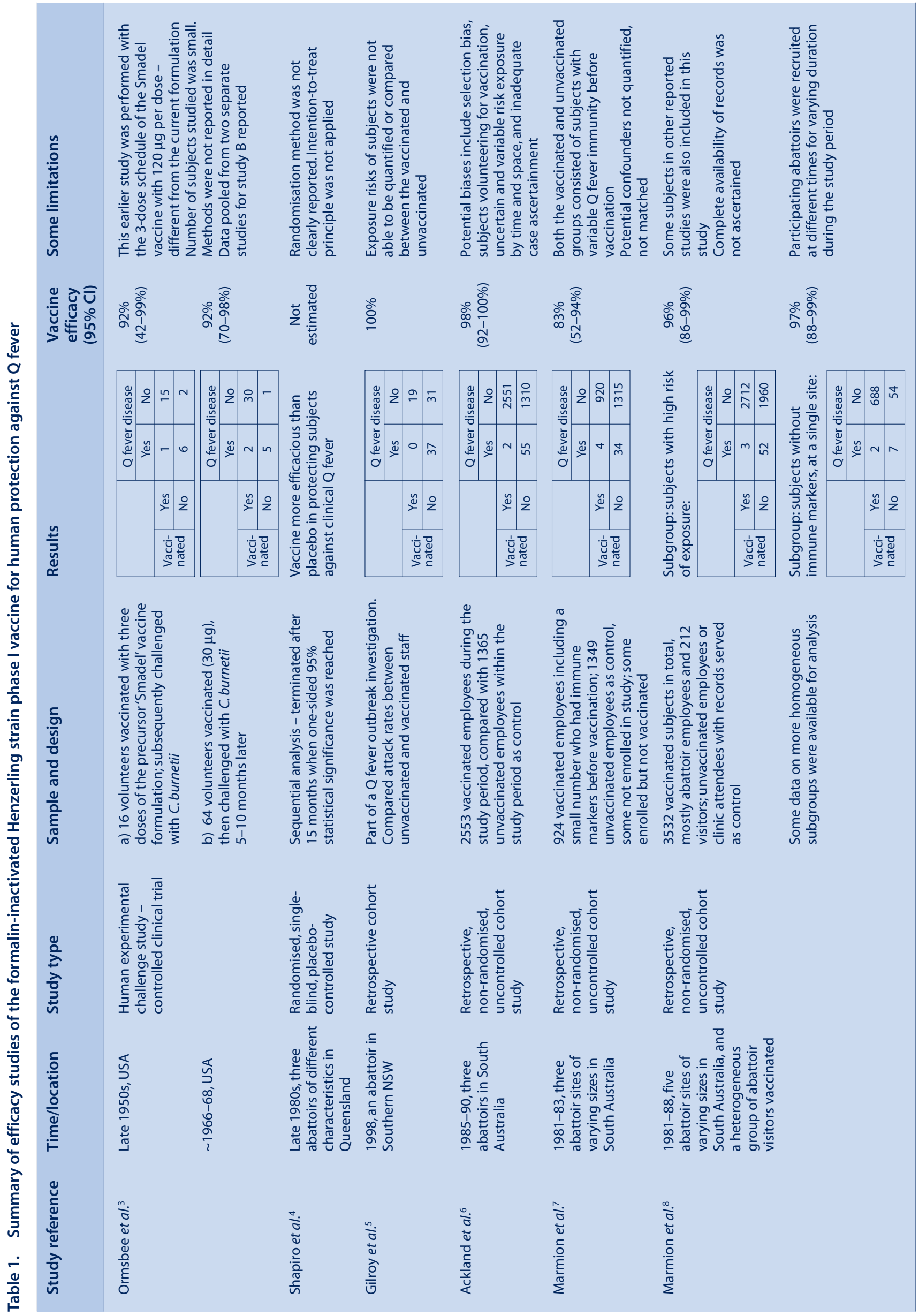


vaccination program and assumed a vaccine efficacy of $98 \%$. It concluded on the basis of direct and indirect costs resulting from Workers' Compensation claims, that application of $Q$ fever vaccine in high risk occupational settings would result in cost savings from a societal perspective. ${ }^{9}$ Extrapolating 1993-94 data from NSW, an Australian review of Q fever conservatively estimated that the disease costs Australia approximately A $\$ 1$ million and more than 1700 weeks of work time lost annually. ${ }^{10}$

\section{Discussion}

Although no large-scale, double-blind, randomised, placebo-controlled studies have evaluated the Australian registered Q fever vaccine, and despite the obvious limitations in the design of published studies, which mean that no precise measure of vaccine efficacy is available, study findings consistently suggest that vaccine efficacy is high compared with other vaccines currently used in Australian public health programs (eg, greater than $80 \%$ for pertussis vaccine, and $\sim 30-80 \%$ for influenza vaccine for older people, depending on the selected outcome indicator). Although uncertainty with respect to the lower efficacy confidence limit may constrain decisions regarding the vaccine's application in lower risk situations, in established high-risk environments, particularly of an occupational nature, it has considerable protective value.

It is important that ongoing availability of an effective Q fever vaccine be ensured for protecting high-risk groups.

\section{Acknowledgements}

Data from the NSW Notifiable Diseases Database was accessed through the Health Outcomes Information and Statistics Toolkit (HOIST) system, a data access, analysis and reporting facility established and operated by the Centre for Epidemiology and Research, Population Health Division, NSW Department of Health

\section{References}

1. Maurin M, Raoult D. Q fever. Clin Microbiol Rev 1999; 12: 518-53.

2. Grieg JE, Patel MS, Clements MS, Taylor NK. Control strategies for $\mathrm{Q}$ fever based on results of pre-vaccination screening in Victoria, 1998 to 2001. Aust N Z J Public Health 2005; 29: 53-7.

3. Ormsbee RA, Marmion BP. Prevention of Coxiella burnetii infection: vaccines and guidelines for those at risk. In: Marrie TJ, editor. Q fever: the disease. Volume 1. Chapter 12. Boca Raton, Florida: CRC Press, 1990.

4. Shapiro RA, Siskind V, Schofield FD, Stallman N, Worswick DA, Marmion BP. A randomized, controlled, doubleblind, cross-over, clinical trial of Q fever vaccine in selected Queensland abattoirs. Epidemiol Infect 1990; 104: 267-73.

5. Gilroy N, Formica N, Beers M, Egan A, Conaty S, Marmion B. Abattoir-associated Q fever: a $\mathrm{Q}$ fever outbreak during a Q fever vaccination program. Aust N Z J Public Health 2001; 25: $362-7$.

6. Ackland JR, Worswick DA, Marmion BP. Vaccine prophylaxis of Q fever. A follow-up study of the efficacy of Q-Vax (CSL) 1985-1990. Med J Aust 1994; 160: 704-8.

7. Marmion BP, Kyrkou M, Worswick D, Esterman A, Ormsbee RA, Wright J, Cameron S, Feery B, Collins W. Vaccine prophylaxis of abattoir-associated Q fever. Lancet 1984; 342: 1411-4. doi:10.1016/S0140-6736(84)91617-9

8. Marmion BP, Ormsbee RA, Kyrkou M, Wright J, Worswick DA, Izzo AA, Esterman A, Feery B, Shapiro RA. Vaccine prophylaxis of abattoir-associated Q fever: eight years' experience in Australian abattoirs. Epidemiol Infect 1990; 104: 275-87.

9. Kermode M, Yong K, Hurley S, Marmion B. An economic evaluation of increased uptake in $\mathrm{Q}$ fever vaccination among meat and agricultural industry workers following implementation of the National Q Fever Management Program. Aust N Z J Public Health 2003; 27: 390-8.

10. Garner MG, Longbottom HM, Cannon RM, Plant AJ. A review of Q fever in Australia 1991-1994. Aust N Z J Public Health 1997; 21: 722-30. 